\title{
A Note on Three-Dimensional Scattering and Diffraction by a Hemispherical Canyon, II: Normal Incident P- Point Source
}

\author{
Guanying Zhu and Vincent W Lee* \\ USC Viterbi School of Engineering, USA
}

Submission: August 04, 2017; Published: October 27, 2017

*Corresponding author: Vincent W Lee, USC Viterbi School of Engineering, USA, Email: vlee@usc.edu

\begin{abstract}
This paper revisits the boundary-valued problem of the three-dimensional (3-D) scattering and diffraction of seismic spherical waves by a hemispherical canyon in an elastic half-space, a problem of interest to both earthquake engineers and strong-motion seismologists. The analytical solution of normal incidence of plane P-wave (axi symmetric) has recently been presented in [1], using the half-range expansion of odd-order Legendre polynomials in terms of even-order Legendre polynomials. The use of excitation by plane harmonic waves is common in many studies of the amplification phenomena by surface and subsurface topographies. It is assumed and reasoned that when the spherical (3-D) and/or cylindrical (2-D) wave fronts are sufficiently far from the earthquake source, such plane wave approximation is adequate. The case of 2-D SH-wave source was recently studied by [2]. The results are compared with that from plane wave incidence. It concluded that the plane-wave approximation did provide sufficient and reasonable approximation. This paper now uses a 3-D point source vertically below a hemispherical canyon and studies how the results are when compared to that of vertical plane wave incidence.
\end{abstract}

Keywords: 3-D point source; Hemispherical canyon; Elastic half-space

\section{Introduction}

As was discussed previously in [1], the odd Legendre polynomial of half-range expansion can effectively solve for the waves scattered around a 3-D hemispherical canyon in a elastic, homogenous, and isotropic half-space with a normal plane P-wave incidence. In this paper, the same methodology will be applied to a similar model of canyon but the incidence is now a point-sourced P-wave.

In the perspective of seismic technology, a point-sourced incidence is very pertinent for an earth-quake with a shallow epicenter. For this, some numerical approaches were developed, such as the boundary element method by [2-4], and the finite difference scheme by [5]. An analytic method of wave diffraction around a small full-space sphere was obtained by [6], in which the method of the image of a point source developed by [7] was borrowed. Nevertheless, no effective analytic solutions for the P- or SV-incidence in the half-space have been accomplished, because of the difficulties of the mode conversion.

Recently in 2013, [2] investigated the finite line source at a distance comparable to the size of the circular alluvial valley for the out-of-plane SH waves. They compared the resultant displacement amplitudes with those by the plane SH-incidence and provided a positive conclusion that the plane waves are good approximations for studies of earthquake engineering. In the end of this paper, the same conclusion will be made based on the comparison of results by the plane P-wave incidence and by the point-source with various frequencies.

\section{The Model}

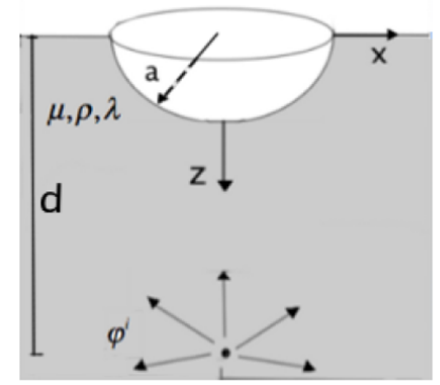

Figure 1: 3-D hemispherical canyon with point-source incidence.

In Figure 1, a hemispherical canyon of radius a is located on the surface of the half-space, which is featured by its density $\rho$, and Lam'e's constants $\mu$, and $\lambda$. An incident point 
source situates below the center of the canyon, and is emitting longitudinal waves (P-wave) only. The distance from the point source to the canyon center is $d$, which is perpendicular to the half-space surface (Figure 1).

The spherical and rectangular Cartesian coordinates centering at the canyon center are adopted. Figure 2 shows the relationship between the Cartesian and the spherical systems. The $x-y$ plane is the half-space surface, and following the righthand-rule, the $\mathrm{z}$-axis is pointing downward. The spherical coordinates is composed by the radial distance $r$, the polar angle , and the azimuthal angle $\phi \theta$. in this case is only half-ranged $\theta \in\left[0, \frac{\pi}{2}\right]$, and $\theta=\frac{\pi}{2}$ on the half-space surface (Figure 2).

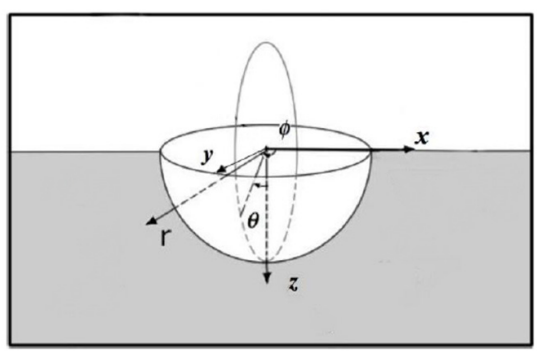

Figure 2: The spherical and Cartesian coordinates.

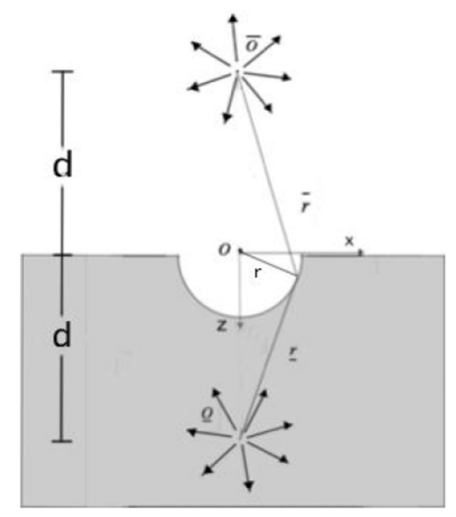

Figure 3: The spherical coordinate system for the point-source $P$-incidence and its image.

In order to describe the point-source with P-wave potential , $\varphi^{(i)}$ a spherical system originated at the point source center is also selected. In Figure 3, 0 refers to the center of this new system of depth $d$ vertically below the center of the canyon, and $r$ is the radial distance measured from 0 . The incident potential $\varphi^{(i)}$ with harmonic frequency can then be expressed as:

$$
\varphi^{(i)}=\varphi_{0} \frac{\exp \left(i k_{\alpha} r-i \omega t\right)}{r}=i \varphi_{0} k_{\alpha} h_{0}^{(1)}\left(k_{\alpha} r\right) \exp (-i \omega t)
$$

where $\varphi_{0}$ is a scale factor, $k_{\alpha}=\frac{\omega}{C_{\alpha}}$, with $C_{\alpha}$ the P-wave speed, is the wave number of the P-wave, and $h_{0}^{(1)}$ is Hankel function of the first kind of order 0 for that the incident wave is symmetrically outward propagating (Figure 3).

From Graf's Addition Theorem [8] and [9], $\varphi^{(i)}$ in (1) can be expanded in terms of the $(r, \theta, \phi)$ coordinates with origin at the canyon center in the following form (with the time factor $\exp (-i \omega t)$ omitted):

$$
i \varphi_{0} k_{\alpha} h_{0}^{(1)}\left(k_{\alpha} r\right)=\left\{\begin{array}{l}
\sum_{n=0}^{\infty} i \varphi_{0} k_{\alpha}(2 n+1) h_{n}^{(1)}\left(k_{\alpha} d\right) j_{n}\left(k_{\alpha} r\right) P_{n}(-u), r<d ; \\
\sum_{n=0}^{\infty} i \varphi_{0} k_{\alpha}(2 n+1) j_{n}\left(k_{\alpha} d\right) h_{n}^{(1)}\left(k_{\alpha} r\right) P_{n}(-u), r \geq d ;
\end{array}\right.
$$

where $j_{n}$ and $h_{n}^{(1)}$ are respectively spherical Bessel function and Hankel function both of the first kind of order $\mathrm{n}$, and $P_{n}$ with argument $u=\cos \theta(0 \leq u \leq 1)$ is the Legendre polynomial of order $\mathrm{n}$. Note that the argument of $P_{n}$ is $-u$, instead of $\mathrm{u}$, because the z-axis is pointing downward. Illuminated by the image methods used in related 2D papers previously, such as [10], the reflected wave potential due to the half-space boundary here can now be represented by a negative (anti-symmetric) source $\varphi^{(r)}$ with respect to $\varphi^{(i)}$ relative to the half-space surface. In [10], for SH wave, a positive (symmetric) image source was used. The 0 in Fig. 3 indicates the source center of the reflected wave of distance $\mathrm{d}$ vertically above the center of the canyon. Here, another polar system originated at $\mathrm{O}$ is adopted for the expression of , $\varphi^{(r)}$ then

$$
\varphi^{(r)}=-\varphi_{0} \frac{\exp \left(i k_{\alpha} \bar{r}-i w t\right)}{\bar{r}}=-i \varphi_{0} k_{\alpha} h_{0}^{(1)}\left(k_{\alpha} \bar{r}\right) \exp (-i \omega t),
$$

Where $r$ is the radial distance from 0 . The time factor $\exp (-i \omega t)$ will again be omitted with the steady-state assumption. The Graf's Addition Theorem is again applied to (3) to obtain the expression of $\varphi^{(r)}$ by the spherical coordinates $(r, \theta, \phi)$ in the form of

$$
-i \varphi_{0} k_{\alpha} h_{0}^{(1)}\left(k_{\alpha} \bar{r}\right)=\left\{\begin{array}{l}
\sum_{n=0}^{\infty}-i \varphi_{0} k_{\alpha}(2 n+1) h_{n}^{(1)}\left(k_{\alpha} d\right) j_{n}\left(k_{\alpha} r\right) P_{n}(u), r<d ; \\
\sum_{n=0}^{\infty}-i \varphi_{0} k_{\alpha}(2 n+1) j_{n}\left(k_{\alpha} d\right) h_{n}^{(1)}\left(k_{\alpha} r\right) P_{n}(u), r \geq d ;
\end{array}\right.
$$

The stress-free boundary conditions on the half-space surface are to be examined, to verify that $\varphi^{(r)}$ is the reflected wave of $\varphi^{(i)}$ in the half-space. These conditions at $\mathrm{z}=0$, are

$$
\sigma_{z z}=T_{z x}=T_{z y}=0,
$$

Which should be satisfied by the summation of $\varphi^{(i)}$ and $\varphi^{(r)}$. In the spherical coordinate system, where $\phi$-term is automatically satisfied due to the property of axisymmetric for this model so that the conditions in (5) can be expressed as

$$
\left.\sigma_{\theta}\right|_{\theta=\frac{\Pi}{2}}=\left.\tau_{\theta r}\right|_{\theta=\frac{\Pi}{2}}=0 .
$$

To superimpose $\varphi^{(i)}$ and $\varphi^{(r)}$, the total wave '(f f) can be expressed as $\varphi^{(f f)}=\varphi^{(i)}+\varphi^{(r)}=\left\{\begin{array}{cc}\sum_{n=1,3,5,}^{\infty} & a_{n}^{(r<d)} j_{n}\left(k_{\alpha} r\right) P_{n}(u), r<d ; \\ \sum_{n=1,3,5, \ldots,}^{\infty} & a_{n}^{(r 2 d)} h_{n}^{(i)}\left(k_{\alpha} r\right) P_{n}(u), r \geq d ;\end{array}\right.$

where $a_{n}^{(r<d)}=-2(2 n+1) i \varphi_{0} k_{\alpha} h_{n}^{(1)}\left(k_{\alpha} d\right) n=1,3,5$,

$$
a_{n}^{(r \geq d)}=-2(2 n+1) i \varphi_{0} k_{\alpha} j_{n}\left(k_{\alpha} d\right) \quad n=1,3,5,
$$




\section{The Free-field Potential}

Now it is going to show that $\varphi^{(f f)}$ naturally satisfies both half-space boundary conditions in (6).

The zero normal stress boundary condition on the half-space surface $\left.\cdot \sigma_{\theta}\right|_{\theta=\frac{\Pi}{2}}=0$

From [8], when $\mathrm{r}<\mathrm{d}$

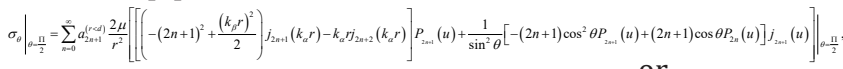

$\left.\sigma_{\theta}\right|_{\theta=\frac{\Pi}{2}}=\sum_{n=0}^{\infty} a_{2 n+1}^{(r<d)} \frac{2 \mu}{r^{2}}\left[\left(-(2 n+1)^{2}+\frac{\left(k_{\beta} r\right)^{2}}{2}\right) j_{2 n+1}\left(k_{\alpha} r\right)-k_{\alpha} r j_{2 n+2}\left(k_{\alpha} r\right)\right] P_{2 n+1}$

Similarly, when $\mathrm{r} \geq \mathrm{d}$,

$\left.\sigma_{\theta}\right|_{\theta=\frac{\Pi}{2}}=\sum_{n=0}^{\infty} a_{2 n+1}^{(r \alpha d)} \frac{2 \mu}{r^{2}}\left[\left(-(2 n+1)^{2}+\frac{\left(k_{\beta} r\right)^{2}}{2}\right) h_{2 n+1}^{(1)}\left(k_{\alpha} r\right)-k_{\alpha} r h_{2 n+1}^{(1)}\left(k_{\alpha} r\right)\right] P_{2 n+1}(0)$.

Knowing $P_{2 n+1}(0) \equiv 0$ that , the boundary condition $\left.\sigma_{\theta}\right|_{\theta=\frac{\pi}{2}}=0$ is always automatically satisfied.

The zero shear stress boundary condition on the halfspace surface.

The general relationship between the longitudinal wave potential and the shear stress is

$\tau_{r \theta}=\frac{2 \mu}{r}\left(\frac{\partial^{2} \varphi}{\partial r \partial \theta}-\frac{\partial \varphi}{r \partial \theta}\right)$

The equations in (7) will be re-expressed in terms of the even Legendre polynomials, because all boundary conditions are in half-, not full-, space. Such technique is already discussed [1].

From [1]:

$P_{2 m+1(u)}=\sum_{n=0}^{\infty} \gamma_{m n} P_{2 n}(u)$,

Where [1]: $\gamma_{m n}=\frac{\int_{0}^{1} P_{2 m+1}(u) P_{2 n}(u) d u}{\int_{0}^{1}\left(P_{2 n}(u)\right)^{2} d u}=\frac{(4 n+1)(-1)^{(m+n+1)}(2 m+1) !(2 n) !}{2^{(m+n)}(2 n-2 m+1)(2 n+2 m+2)(n !)^{2}(m !) 2}$

Then (7) becomes

$$
\varphi^{(f f)}=\left\{\begin{array}{l}
\sum_{n=0}^{\infty} \sum_{m=0}^{\infty} \gamma_{m n} a_{2 m+1}^{(r<d)} j_{2 m+1}\left(k_{\alpha} r\right) P_{2 n}(u), r<d ; \\
\sum_{n=0}^{\infty} \sum_{m=0}^{\infty} \gamma_{m n} a_{2 m+1}^{(r \geq d)} h_{2 m+1}^{(1)}\left(k_{\alpha} r\right) P_{2 n}(u), r \geq d .
\end{array}\right.
$$

This new expression gives

$$
\frac{\partial \varphi^{(f f)}}{\partial \theta}=\left\{\begin{array}{l}
\sum_{n=0}^{\infty} \sum_{m=0}^{\infty} \gamma_{m n} a_{2 m+1}^{(r<d)} j_{2 m+1}\left(k_{\alpha} r\right) P_{2 n}^{1}(u), r<d ; \\
\sum_{n=0}^{\infty} \sum_{m=0}^{\infty} \gamma_{m n} a_{2 m+1}^{(r \geq d)} h_{2 m+1}^{(1)}\left(k_{\alpha} r\right) P_{2 n}^{1}(u), r \geq d ;
\end{array}\right.
$$

Where $P^{1}(u)$ is the first derivative of the Legendre polynomial with order $2 \mathrm{n}$ with respect to $\theta$-i.e. $P_{2 n}^{1}(u)=\frac{\partial P_{2 n}^{1}(u)}{\partial \theta}$.

From (11) and (14), $\left.\tau_{r \theta}\right|_{\theta=\frac{\pi}{2}}=0$ due to $\varphi^{(f f)}$ is

$$
\left.\tau_{r \theta}\right|_{\theta=\frac{\Pi}{2}}=\left\{\begin{array}{l}
\frac{2 \mu}{r} \sum_{n=0}^{\infty} \sum_{m=0}^{\infty} \gamma_{m n} a_{2 m+1}^{(r<d)} j_{2 m+1}\left(\frac{\partial j_{2 m+1}\left(k_{\alpha} r\right)}{\partial r}-\frac{1}{r}\right) P_{2 n}^{1}(u), r<d ; \\
\frac{2 \mu}{r} \sum_{n=0}^{\infty} \sum_{m=0}^{\infty} \gamma_{m n} a_{2 m+1}^{(r<d)}\left(\frac{\partial h_{2 m+1}^{(1)}\left(k_{\alpha} r\right)}{\partial r}-\frac{1}{r}\right) P_{2 n}^{1}(u), r \geq d .
\end{array}\right.
$$

Since $P_{2 n}^{1}(0) \equiv 0$ at $\theta=\frac{\pi}{2}$ for the half-space surface, the shear stress boundary condition $\left.\tau_{r \theta}\right|_{\theta=\frac{\Pi}{2}}=0$ is again automatically satisfied.
Therefore $\varphi^{(r)}$ can be verified to be the reflected wave from the half-space (without any topography). In addition, the $\varphi^{(f f)}$ defined in (7) is the free-field wave for a point-source P-incidence in the half-space.

\section{The Scattered Waves}

The scattered waves are generated in the presence of the hemispherical canyon. The same methodology is applied as in [1] that the scattered wave's potentials will again be expressed in terms of odd Legendre polynomials only. So that

$$
\begin{aligned}
& \varphi^{(s)}=\sum_{n=0}^{\infty} A_{2 n+1} h_{2 n+1}^{(1)}\left(k_{\alpha} r\right) P_{2 n+1}(u), \\
& \chi^{(s)}=\sum_{n=0}^{\infty} C_{2 n+1} h_{2 n+1}^{(1)}\left(k_{\beta} r\right) P_{2 n+1}(u),
\end{aligned}
$$

Where $k_{\beta}=\frac{\omega}{C_{\beta}}$ is the shear wave number, with $C_{\beta}$ the shear wave speed in the half-space. From (12a), the wave potentials can also be expressed in terms of the Legendre polynomials of even degrees as

$$
\begin{aligned}
& \varphi^{(s)}=\sum_{m=0}^{\infty}\left(\sum_{n=0}^{\infty} A_{2 m+1} h_{2 m+1}^{(1)}\left(k_{\alpha} r\right) \gamma_{m n}\right) P_{2 n}(u), \\
& \chi^{(s)}=\sum_{n=0}^{\infty}\left(\sum_{n=0}^{\infty} C_{2 m+1} h_{2 m+1}^{(1)}\left(k_{\beta} r\right) \gamma_{m n}\right) P_{2 n}(u),
\end{aligned}
$$

The summation of all the waves $\varphi^{\left.(f)^{2}\right)}, \varphi^{(s)}$, and $\chi^{(s)}$ need to satisfy all boundary conditions on both the half-space surface and the hemispherical canyon surface, which are

$$
\begin{aligned}
& \left.\sigma_{\theta}\right|_{\theta=\frac{\pi}{2}}=\left.\tau_{r \theta}\right|_{\theta=\frac{\Pi}{2}}=0, \quad(18) \\
& \text { and } \\
& \left.\sigma_{r}\right|_{r=a}=\left.\tau_{r \theta}\right|_{r=a}=0 .
\end{aligned}
$$

All boundary conditions will be discussed as follows:

\section{The zero normal stress boundary condition on the half-space surface . $\left.\sigma_{\theta}\right|_{\theta=\frac{\Pi}{2}}=0$}

Only the scattered P- and S-wave potentials will be taken into consideration when evaluating normal stresses since $\varphi^{(f)}$ is already shown to satisfy this boundary condition. With (16), the $\mathrm{P}$ - and S- potentials with argument $u=\cos \theta$ have the following expressions of the normal stress on the half-space surface [1]:

$$
\left.\sigma_{\theta}\right|_{\theta=\frac{\pi}{2}}=\sum_{n=0,1,2,2}^{\infty}\left[A_{2 n+1} E_{21}^{(3)}\left(2 n+1, k_{\alpha} r\right)+C_{2 n+1} E_{23}^{(3)}\left(2 n+1, k_{\beta} r\right)\right] P_{2 n+1}(0)
$$

Equation (20) is the summation over all the odd integers, $\mathrm{n}=1,3,5, \ldots$, and $P_{n}(0)=0$ for all $r \geq\left. a \quad \sigma_{\theta}\right|_{\theta=\frac{\pi}{2}}=0$ odd n-degree Legendre polynomials, and thus for all , is always automatically satisfied. All the $\mathrm{E}_{\mathrm{ij}}$ functions above and following in the paper are listed in Appendix A.

\section{The zero shear stress boundary condition on the half- space surface .}

As in Boundary Condition 1, only the scattered P- and S-wave potentials are used to satisfy the shear stress on half-space surface. Here the expressions in (17) are adopted, and then the shear stress by the P- and S-waves is expressed as [1]: 
$\left.\tau_{\theta r}\right|_{\theta=\frac{\pi}{2}}=\sum_{\substack{n \geq m, n \\ n=1,2,3}}^{\infty}\left[\sum_{m=0,0,1,2,}^{\infty}\left(A_{2 m+1} E_{41}^{(3)}\left(2 m+1, k_{\alpha} r\right)+C_{2 m+1} E_{43}^{(3)}\left(2 m+1, k_{\beta} r\right) \gamma_{m n}\right) P_{2 n}^{1}(0)\right.$

$P_{n}^{1}(0)$ Is the first derivative of $P_{n}(0)$ so that $P_{n}^{1}(0)$ is odd when $\mathrm{n}$ is even? Summing up along $\mathrm{n}=1,2,3, \ldots$, the result is definitely zero since every term is multiplying an odd function with argument zero. Therefore conclusion could be reached that the zero shear stress is also satisfied on the half-space surface. Now two out of the four boundary conditions are automatically satisfied (removed), so then the remaining two boundary conditions will next be utilized to solve for the two sets of unknown coefficients, $\left\{A_{2 n+1}\right\}$ and $\left\{C_{2 n+1}\right\}$ for $\mathrm{n}=0,1,2, \ldots$, of the $\mathrm{P}$ - and S-wave potentials.

\section{The zero normal stress boundary condition on the canyon surface .}

The total normal stress $\sigma_{r}$ is composed of the free-field wave and the scattered waves that

$$
\left.\sigma_{r}\right|_{r=a}=\left.\sigma_{r}^{(f f)}\right|_{r=a}+\left.\sigma_{r}^{(s)}\right|_{r=a}=0
$$

Thus the following relationship is then obtained:

$$
\left.\sigma_{r}^{(s)}\right|_{r=a}=-\left.\sigma_{r}^{(f f)}\right|_{r=a} \text {. }
$$

Where $\sigma_{r}^{(f f)}$ is the normal stress due to the free-field potential, and $\sigma_{r}^{(s)}$ is that due to the scattered waves.

Their expressions in the spherical coordinate system are given as [1]:

$$
\begin{aligned}
& \left.\sigma_{r}^{(f f)}\right|_{r=a}=\sum_{n=0}^{\infty} a_{2 n+1} E_{11}^{(1)}\left(2 n+1, k_{\alpha} \mathrm{a}\right) \mathrm{P}_{2 n+1}(u), \\
& \left.\sigma_{r}^{(s)}\right|_{r=a}=\sum_{n=0}^{\infty}\left(A_{2 n+1} E_{11}^{(3)}\left(2 n+1, k_{\alpha} \mathrm{a}\right)+C_{2 n+1} E_{13}^{(3)}\left(2 n+1, k_{\beta} \mathrm{a}\right)\right) \mathrm{P}_{2 n+1}(u),
\end{aligned}
$$

Combining (23) and (24), there is

$$
\sum_{n=0}^{\infty}\left(A_{2 n+1} E_{11}^{(3)}\left(2 n+1, k_{a} \mathrm{a}\right)+C_{2 n+1} E_{13}^{(3)}\left(2 n+1, k_{\beta} \mathrm{a}\right)\right) \mathrm{P}_{2 n+1}(u)=-\sum_{n=0}^{\infty} a_{2 n+1} E_{11}^{(1)}\left(2 n+1, k_{a} \mathrm{a}\right) \mathrm{P}_{2 n+1}(u) \text {. }
$$

Then to apply the orthogonality of odd Legendre polynomials over the half-space, (25) is simplified to be, for $n=0,1,2, \ldots$.

$$
A_{2 n+1} E_{11}^{(3)}\left(2 n+1, k_{\alpha} \mathrm{a}\right)+C_{2 n+1} E_{13}^{(3)}\left(2 n+1, k_{\beta} \mathrm{a}\right)=-a_{2 n+1} E_{11}^{(1)}\left(2 n+1, k_{\alpha} \mathrm{a}\right) .
$$

The zero shear stress boundary conditions on the canyon surface $\left.\cdot \tau_{r \theta}\right|_{r=a}=0$

As in to Boundary Condition 3, the zero shear stress $\left.\tau_{r \theta}\right|_{r=a}$ is from both the free-field wave and the scattered waves, so that [1]:

$$
\left.\tau_{r \theta}^{(s)}\right|_{r=a}=-\left.\tau_{r \theta}^{(f f)}\right|_{r=a},
$$

Where $\tau_{r \theta}^{(s)}, \tau_{r \theta}^{(f f)}$ are respectively the shear stresses due to the scattered waves and the free-field waves. Here

$$
\begin{aligned}
& \left.\tau_{r \theta}^{(f i)}\right|_{r=a}=\sum_{n=0}^{\infty} a_{2 n+1} E_{t i 1}^{(i)}\left(2 n+1, k_{\alpha} \mathrm{a}\right) \mathrm{P}_{2 a+1}^{1}(u) \text {. } \\
& \left.\left.\tau_{r \theta}^{(s)}\right|_{r=a}=\sum_{n=0}^{\infty}\left(A_{2 n+1} E_{4+1}^{(3)}\left(2 n+1, k_{\alpha} \mathrm{a}\right)+C_{2 n+1} E_{43}^{(3)}\left(2 n+1, k_{\beta} \mathrm{a}\right)\right)\right)_{2}^{!}(u) \text {. }
\end{aligned}
$$

Equations (27) and (28) give

$$
\sum_{n=0}^{\infty}\left(A_{2 n+1} E_{41}^{(3)}\left(2 n+1, k_{\alpha} \mathrm{a}\right)+C_{2 n+1} E_{43}^{(3)}\left(2 n+1, k_{\beta} \mathrm{a}\right)\right) \mathrm{P}_{2 n+1}^{1}(u)=-\sum_{n=0}^{\infty}\left(a_{2 n+1} E_{41}^{(1)}\left(2 n+1, k_{\alpha} \mathrm{a}\right)\right) \mathrm{P}_{2 n+1}^{1}(u)
$$

After applying the orthogonality of $\left\{\mathrm{P}_{2 n+1}^{1}(u)\right\}, n=0,1,2, \ldots \ldots$. , that is a set of even Legendre functions, for each $\mathrm{n}$ the coefficients $A_{2 n+1}$ and $C_{2 n+1}$ have the following relation, for $\mathrm{n}=0,1,2, \ldots$.

$A_{2 n+1} E_{41}^{(3)}\left(2 n+1, k_{\alpha} \mathrm{a}\right)+C_{2 n+1} E_{43}^{(3)}\left(2 n+1, k_{\beta} \mathrm{a}\right)=-a_{2 n+1} E_{11}^{(1)}\left(2 n+1, k_{\alpha} \mathrm{a}\right)$

(26) And (30) can be rearranged to be: for $n=0,1,2, \ldots$

$\left[\begin{array}{ll}E_{11}^{(3)}\left(2 n+1, k_{\alpha} \mathrm{a}\right) & E_{13}^{(3)}\left(2 n+1, k_{\beta} \mathrm{a}\right) \\ E_{41}^{(3)}\left(2 n+1, k_{\alpha} \mathrm{a}\right) & E_{43}^{(3)}\left(2 n+1, k_{\beta} \mathrm{a}\right)\end{array}\right]\left\{\begin{array}{l}A_{2 n+1} \\ C_{2 n+1}\end{array}\right\}=-a_{2 n+1}\left\{\begin{array}{l}E_{11}^{(1)}\left(2 n+1, k_{\alpha} \mathrm{a}\right) \\ E_{41}^{(1)}\left(2 n+1, k_{\alpha} \mathrm{a}\right)\end{array}\right\}$

Therefore, the coefficients $A_{n}$ and $C_{n}$ are given by, for $\mathrm{n}=$ $1,3,5, \ldots$,

$$
\left\{\begin{array}{l}
A_{n} \\
C_{n}
\end{array}\right\}=\frac{-a_{n}}{\operatorname{Det}(n)}\left[\begin{array}{cc}
E_{43}^{(3)}\left(n, k_{\beta} \mathrm{a}\right) & -E_{13}^{(3)}\left(n, k_{\beta} \mathrm{a}\right) \\
-E_{41}^{(3)}\left(n, k_{\alpha} \mathrm{a}\right) & E_{11}^{(3)}\left(n, k_{\alpha} \mathrm{a}\right)
\end{array}\right]\left\{\begin{array}{c}
E_{11}^{(1)}\left(n, k_{\alpha} \mathrm{a}\right) \\
E_{41}^{(1)}\left(n, k_{\beta} \mathrm{a}\right)
\end{array}\right\}
$$

With the determinant $\operatorname{Det}(n)=E_{11}^{(3)}\left(n, k_{\alpha} \mathrm{a}\right) E_{43}^{(3)}\left(n, k_{\beta} \mathrm{a}\right)-E_{13}^{(3)}\left(n, k_{\beta} \mathrm{a}\right) E_{41}^{(3)}\left(n, k_{\alpha} \mathrm{a}\right)$. Note that $a_{2 n+1}$ should be $a_{2 n+1}^{(r<d)}$ of (8a) because the source is below the canyon with $r=a<d$.

The free-field coefficients in (32) are

$$
a_{n}=-(4 n+2) i \varphi_{0} k_{\alpha} h_{n}^{(1)}\left(k_{\alpha} d\right) \quad n=1,3,5, \ldots
$$

\section{Surface Displacement}

\section{Vertical Displacements}

The displacement amplitude at a point is a very important assessment of the devastation at that point resulted by the seismic waves. The vertical displacement amplitude $\left|U_{z}\right|$ will be focused here because the problem presented in this paper is an axisymmetric problem with the longitudinal wave from a point source vertically below the canyon. $U_{z}$ Can be calculated from the spherical components $U_{r}$ and $U_{\theta} \quad$ ( $U_{\phi}=0$ from axisymmetry) by the transformation between the two coordinates, where,

$$
U_{z}=U_{r} \cos \theta-U_{\theta} \sin \theta
$$

$$
\begin{aligned}
& \text { With } \\
& U_{r}=\sum_{n=1,3,5, \ldots,}^{\infty}\left[a_{n} D_{11}^{(1)}\left(n, k_{\alpha} \mathrm{a}\right)+A_{n} D_{11}^{(3)}\left(n, k_{\alpha} \mathrm{a}\right)+C_{n} D_{13}^{(3)}\left(n, k_{\beta} \mathrm{a}\right)\right] P_{n}(u), \\
& U_{\theta}=\sum_{n=1,3,5, \ldots,}^{\infty}\left[a_{n} D_{21}^{(1)}\left(n, k_{\alpha} \mathrm{a}\right)+A_{n} D_{21}^{(3)}\left(n, k_{\alpha} \mathrm{a}\right)+C_{n} D_{23}^{(3)}\left(n, k_{\beta} \mathrm{a}\right)\right] \frac{P_{n}(u)}{d \theta}
\end{aligned}
$$

It is noted that, for P-point source, $U_{z}$, the vertical component, is the dominating component of motion. Here $D_{j k}^{(i)}$ are the displacement-potential expressions given in Appendix A.

The displacement amplitude is the magnitude of a complexvalued displacement, so that for $U_{z}$

$$
\left|U_{z}\right|=\left[\operatorname{Re}^{2}\left(U_{z}\right)+\operatorname{Im}^{2}\left(U_{z}\right)\right]^{\frac{1}{2}}(36)
$$

As was stated in (1) that $\varphi_{0}$ is the scale factor for the wave potentials, this scale factor should be determined before proceeding to the numerical calculation. According to the plane P-wave incidence, $\varphi_{0}$ can be determined by the displacement amplitude at the original point such that such that $\left|U_{z}\right|$ resulted by the free-field wave at the canyon center is always " 2 ".

Given $^{\varphi^{(i)}}=\varphi_{0} \frac{\exp ^{i k_{\alpha} r}}{r}$ and $\varphi^{(r)}=-\varphi_{0} \frac{\exp ^{i k_{\alpha} r}}{\bar{r}}$, the vertical displacement due to the incident wave is 


\section{Civil Engineering Research Journal}

$$
U_{z}=\frac{\partial \varphi^{(i)}}{\partial r}=\varphi_{0} \exp \left(i k_{\alpha} r\right)\left[\frac{i k_{\alpha}}{r}-\frac{1}{r^{2}}\right]
$$

And that due to the reflected wave is

$$
U_{z}=\frac{\partial \varphi^{(r)}}{\partial r}=\varphi_{0} \exp \left(i k_{\alpha} \bar{r}\right)\left[\frac{i k_{\alpha}}{\bar{r}}-\frac{1}{\bar{r}^{2}}\right]
$$

Combining (37) and (38), the total vertical displacement at the origin 0 , where $r=\bar{r}=d(\mathrm{r}=0)$, is

$$
\left.U_{z}\right|_{r=0}=2 \varphi_{0} \exp \left(-i k_{\alpha} d\right)\left[\frac{i k_{\alpha}}{d}-\frac{1}{d^{2}}\right]
$$

Therefore, define

$$
\varphi_{0}=\exp \left(-i k_{\alpha} d\right)\left[\frac{i k_{\alpha}}{d}-\frac{1}{d^{2}}\right]^{-1}(40)
$$

So that $\left|U_{z}\right|$ at the center of canyon equals to number " 2 ", and the coefficients of free-field potentials can be expressed as, from $(8 a)$ and $(8 b)$ :

$$
\begin{aligned}
& a_{2 n+1}^{(r<d)}=-(8 n+6) i k_{\alpha} \exp \left(-i k_{\alpha} d\right)\left[\frac{i k_{\alpha}}{d}-\frac{1}{d^{2}}\right]^{-1} h_{2 n+1}\left(k_{\alpha} d\right), \quad n=0,1,2, \ldots . \\
& a_{2 n+1}^{(r r d)}=-(8 n+6) i k_{\alpha} \exp \left(-i k_{\alpha} d\right)\left[\frac{i k_{\alpha}}{d}-\frac{1}{d^{2}}\right]^{-1} j_{2 n+1}\left(k_{\alpha} d\right), n=0,1,2, \ldots .
\end{aligned}
$$

Alternatively, $a_{n}$ in (41a) and (41b) for the calculation of $A_{n}$ and $C_{n}$ is then given by, with $\mathrm{r}=\mathrm{a}<\mathrm{d}$,

$$
a_{n}=\left\{\begin{array}{c}
-(4 n+2) i k_{\alpha} h_{n}^{(1)}\left(k_{\alpha} d\right) \exp \left(-i k_{\alpha} d\right)\left[\frac{i k_{\alpha}}{d}-\frac{1}{d^{2}}\right]^{-1}{ }_{n=1,3,5, \ldots} . \\
0 n=0,2,4, \ldots .
\end{array}\right.
$$

Which contains both the source depth $d$, and the wave number $k_{\alpha}$.

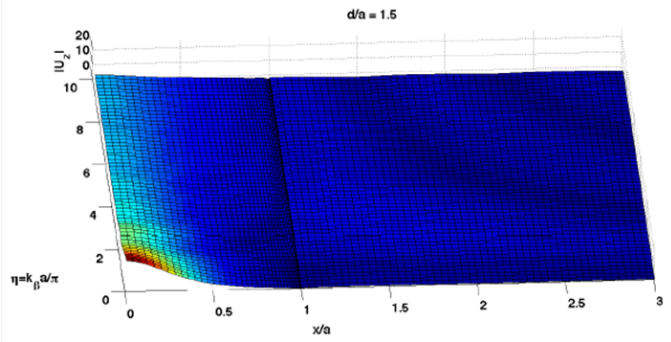

Figure 4: Vertical displacement amplitude $\left|U_{z}\right|$ along $x / a$ for the normal point-source incidence: $\eta=0-10$ and $d=1.5 a$.

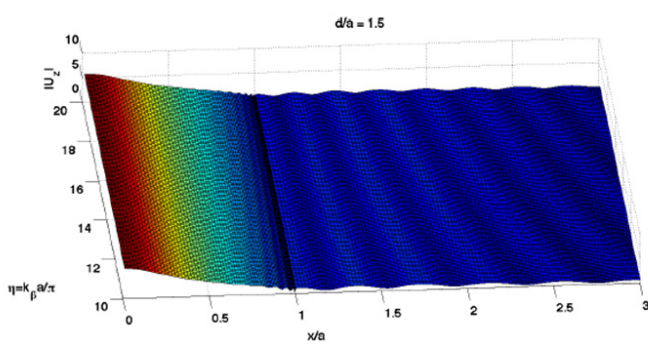

Figure 5: Vertical displacement amplitude $\left|U_{z}\right|$ along $x / a$ for the normal point-source incidence: $\eta=10-20$ and $d=1.5$ a.

Figure 4 to Figure 9 are the vertical displacement amplitudes $\left|U_{z}\right|$ along the dimensionless radial distance (x/a) for a fixed wave source location and various dimensionless frequencies $\eta$, following the definition in [11], where the dimensionless frequency, $\eta$, is used:

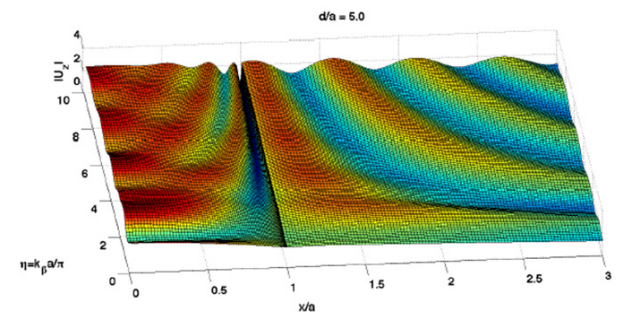

Figure 6: Vertical displacement amplitude $\left|U_{z}\right|$ along x/a for the normal point-source incidence: $\eta=0-10$ and $d=5.0 a$.

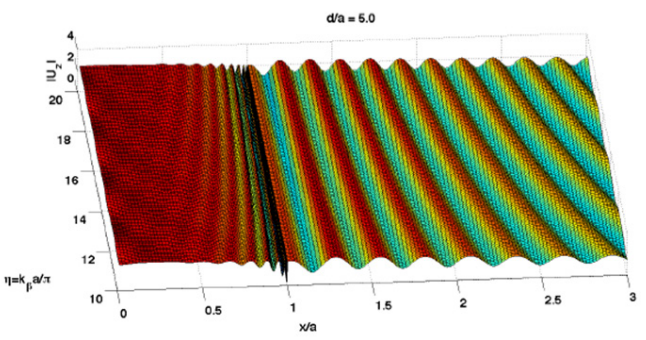

Figure 7: Vertical displacement amplitude $\left|U_{z}\right|$ along x/a for the normal point-source incidence: $\quad \eta=10-20$ and $d=5.0$ a.

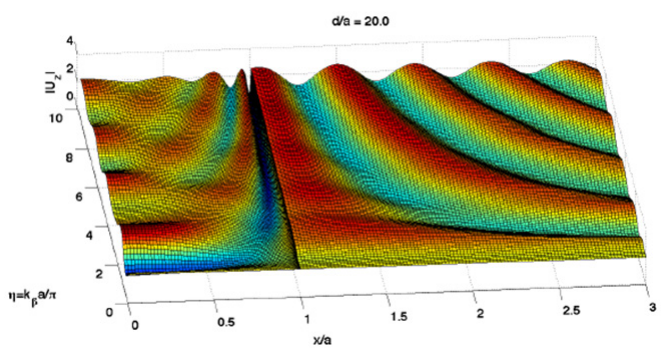

Figure 8: Vertical displacement amplitude $\left|U_{z}\right|$ along $x / a$ for the normal point-source incidence: $\quad \eta=0-10$ and $d=20.0 a$.

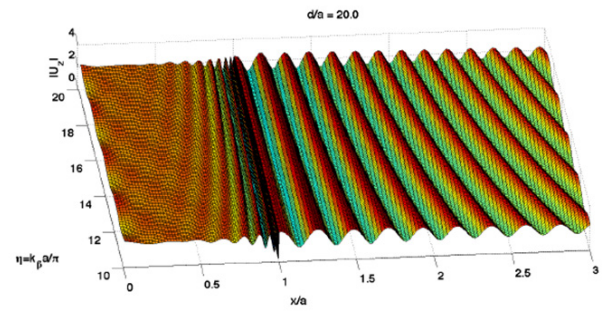

Figure 9: Vertical displacement amplitude $\left|U_{z}\right|$ along x/a for the normal point-source incidence: $\eta=10-20$ and $d=5.0$ a.

$$
\eta=\frac{\omega \mathrm{a}}{\pi \beta}=\frac{2 f \mathrm{a}}{\beta}=\frac{k_{\beta} \mathrm{a}}{\pi}=\frac{2 a}{\lambda_{\beta}}
$$




\section{Civil Engineering Research Journal}

The azimuthal angle $\phi$ will play no part because the model is axisymmetric, the axis $\mathrm{x} / \mathrm{a}$ is the radial distance $\mathrm{r} / \mathrm{a}$ along any direction. $\phi$ The range of $\mathrm{x} / \mathrm{a}$ from 0 to 1 represents the distance from the center to the rim along the canyon surface when goes from $\frac{\pi}{2}$ to 0 . When $x / a>1$, the half-space surface is under discussion.

Figure 4 and Figure 5 are the vertical displacement amplitudes $\left|U_{z}\right|$ respectively from $\eta=0$ to 10 , with the same source depth of $d=1.5$ a. One can see that can be as high as 20 at $\mathrm{x} / \mathrm{a}=0$, the bottommost point of the canyon, when is very small, but it decays quickly along the radial distance. There is little oscillation observable on the half-space surface in the low range when $x / a>1$. With increasing, the magnitude of on the canyon surface levels off to 2 , and there are clear wave patterns on the half-space surface showing that the particles at a relatively far distance from the canyon center start still oscillate.

Figure 6 and Figure 7 are $\left|U_{z}\right|$ respectively from $\eta=0$ to 10 , and from $\eta=10$ to 20 with now source depth of $d=5.0 \mathrm{a}$. This time the maximum in both Figure do not exceed 3.0 from the fact that the source is much farther from the canyon. As in Figure 4 and Figure 5 max gradually levels to 2 as increases. The wave oscillation is now substantial, particularly in the high frequency range. At the rim of the canyon with the ground, where $\mathrm{x} / \mathrm{a}=1$, the" dip and spike" phenomenon, which is described in [1], is also observed.

Figure 8 and Figure 9 contain the more intense wave motions at frequencies of $\eta=0-10$ and $\eta=10-20$ respectively, when the depth of source is at $d=20$ a below the origin. One notes that the amplitude of shaking is more oscillatory, compared with the prior two sets of Figure at $d=1.5 \mathrm{a}$, and $d=5.0 \mathrm{a}$, for a given $\eta$. This is because when the source is deep below the canyon, there is now greater difference in phases and radiation distances for any two points on the half-space surface, resulting in greater differ in the wave amplitudes. At the low frequency range where $\eta$ is close to 0 , the amplitudes along the radial distance are almost constant and this agrees with the observation of the normal plane P-incidence in the previous paper [1]. Moreover, the" dip and spike" phenomena at the rim $(\mathrm{r}=\mathrm{a})$ become more apparent and stable.

Next, Figure 10 consists of $\left|U_{z}\right|$ four plots of at $\mathrm{d} / \mathrm{a}=1.5$ for $=$ $0.02,0.5,1.0$, and 1.5 , respectively. It is apparent that $\left|U_{z}\right|$ at the origin, corresponding to the lowest point of the canyon that is closest to the source decreases with increasing. However, for a location outside the canyon, $\mathrm{r} / \mathrm{a}>1$, its amplitude for $\eta=0.02$ or $\eta=0.5$ are generally smaller than that of $\eta=1$ or $\eta=1.5$. To conclude, for a near source incidence with an equivalently scaled potential, the low incident frequencies accumulate energies at the origin, while at higher frequencies the energy is spread out by the wave propagation. Figure 11 to Figure 13 display the vertical displacement amplitudes $\left|U_{z}\right|$ at $\mathrm{d} / \mathrm{a}=1.5,5$, and 20 for $\eta=0.02,1$, and 20 , respectively.

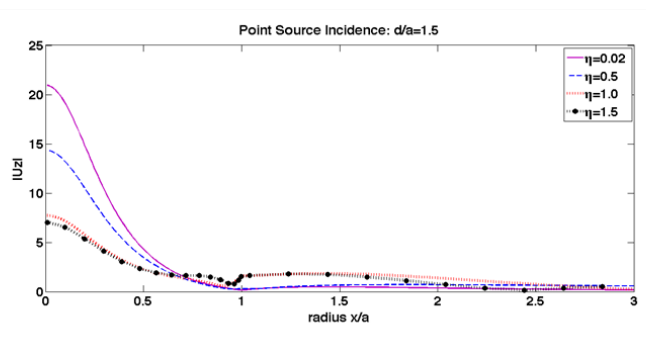

Figure 10: Vertical displacement amplitude along $x / a$ for the normal point-source incidence with $\mathrm{d} / \mathrm{a}=1.5$ and $\eta=0.02,0.5$, 1.0 and 1.5 .

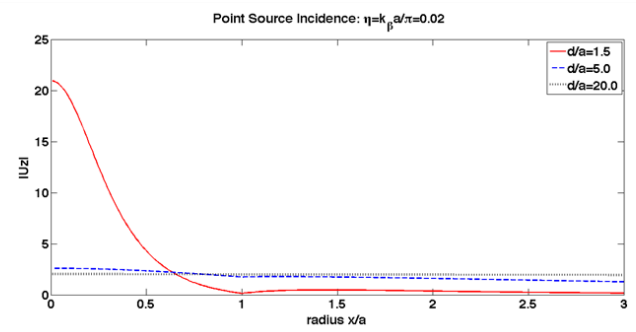

Figure 11: Vertical displacement amplitude along $x / a$ for the normal point-source incidence with $\mathrm{d} / \mathrm{a}=1.5,5$, and 20 for $=\eta$ 0.02

It is shown from Figure 11 that for the very low frequency incidence-i.e. $\eta=0.02$, if the source is shallow ( $d / a=1.5$ here), the bottommost point of the canyon has the most substantial deformation. While on the other hand, if the epicenter is deep, the plots appear to have comparable amplitudes on the halfspace surface outside the canyon to those on the canyon surface.

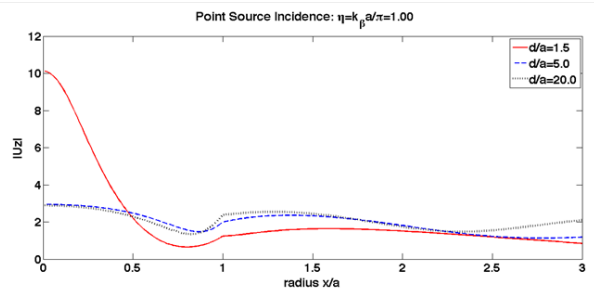

Figure 12: Vertical displacement amplitude along $x / a$ for the normal point-source incidence with $\mathrm{d} / \mathrm{a}=1.5,5$, and 20 for $\eta$ $=1.0$

Figure 12 and Figure 13 show that when $\eta$ gets higher, the canyon bottom (where $\mathrm{x} / \mathrm{a}=0$ ) still has the same high displacement amplitude and hence the same severe damage from a shallow source-at $d / a=1.5$. Unlike the other three Figure at lower frequencies, Figure 13 at $\eta=20.0$ shows that the waves at the canyon and half-space surfaces are more oscillatory, even far from the canyon.

Figure 14 provides $\mathrm{x} / \mathrm{a}=0$ to 100 to investigate the amplitudes in the perspective of a longer range far from the canyon. Apparently, the amplitudes resulted by a shallow wave 


\section{Civil Engineering Research Journal}

source decay faster along the radial distance than that resulted by a deep source. In addition, the amplitudes from all the pointsourced cases will eventually drop to zero, at some points on the half-space surface that is sufficiently far from the canyon.

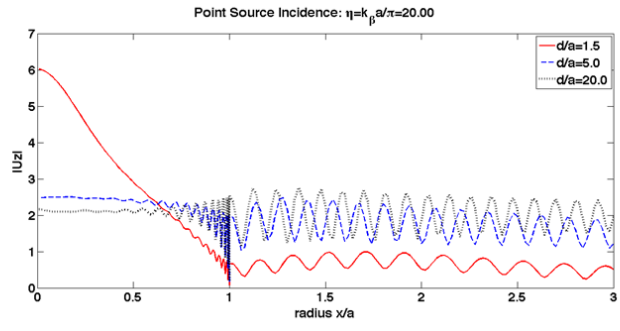

Figure 13: Vertical displacement amplitude along $x / a$ for the normal point-source incidence with $\mathrm{d} / \mathrm{a}=1.5,5$, and 20 for $\eta=$ 20.0

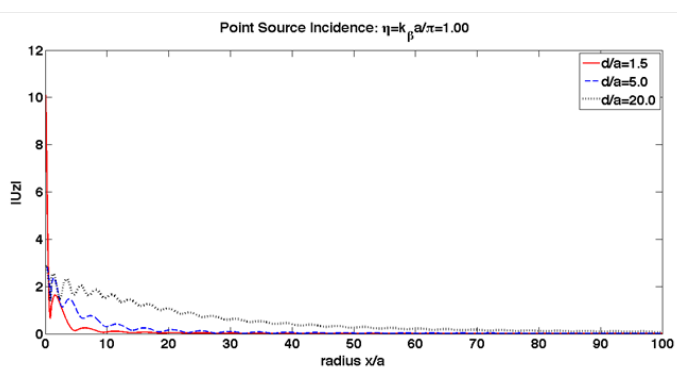

Figure 14: Vertical displacement amplitude along $x / a$ to 100 for the normal point-source incidence with $\mathrm{d} / \mathrm{a}=1.5,5$ and 20 and $=1.0$

\section{Comparison with plane wave incidence: $d>a$}

The plane wave incidences can, intuitively, precisely simulate the point-sourced incidences if the wave sources are deep enough. This sub-section is to examine the conditions for a vertical plane incidence to replace a vertical point-sourced incidence. First of all, the free-field coefficients of a deep normal point-sourced incidence in (33) can be proven equal to the coefficients of the normal plane free-field potentials provided in [1].

From (33), when $d>>$ a is very deep, the coefficients can be simplified as $(\mathrm{n}=0,1,2, \ldots)$ :

$$
a_{2 n+1}=-(8 n+1) i k_{\alpha} \exp (-i k \alpha d)\left[\frac{i k_{\alpha}}{d}\right]^{-1} h_{2 n+1}^{(1)}\left(k_{\alpha} d\right)
$$

While the coefficients for plane wave incidences are [1]:

$a_{2 n+1}=\frac{(8 n+6) i^{(2 n+1)}}{i k_{\alpha}}$

From the mathematical relationship of asymptotic expansion:

$h_{2 n+1}^{(1)}\left(k_{\alpha} d\right) \sim \frac{1}{\left(k_{\alpha} d\right)} e^{\left(k_{\alpha} d+(n+1) \pi\right)}$ when $d \rightarrow \infty$,

(44) and (45) can be shown to be equal.

In Figure 15 to Figure 17, the results of the normal plane incidence and of the normal point-sourced incidences with various depths are visualized and compared. Both the point source and the plane wave are normalized to have unit amplitude displacement at the origin, or center of the canyon, corresponding to free field displacement amplitude of 2 at the half-space surface, after adding the reflected wave. The patterns of amplitudes always agree at the lower range of x/a first closer to the canyon, and then start to differ slightly when the referenced point moves farther away from the canyon. For the case of $=1$ in Figure 15, the plot of $d=100$ a can visually match the result of the plane incidence up to $\mathrm{x} / \mathrm{a}=2.5$; but for $\eta=4$ in Figure 16, the agreeable range shrinks to be around $\mathrm{x} / \mathrm{a}=2$ along the horizontal axis; and for $\eta=8$ in Figure 17, this number of the upper-bound is only less than two. Thus, for a point-sourced incidence at a fixed depth, the high incident frequency narrows the efficient range of a substituted plane incidence.

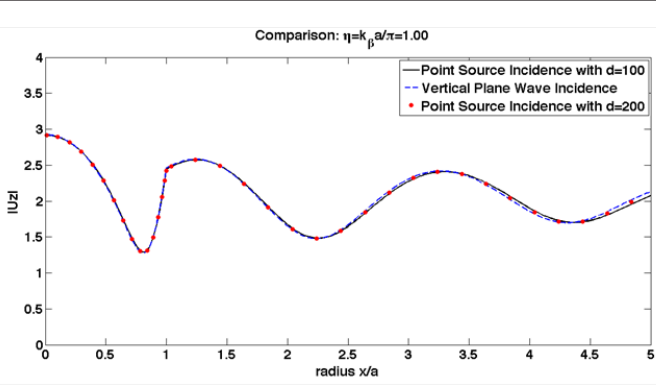

Figure 15: Vertical displacement amplitudes along $\mathrm{x} / \mathrm{a}$ for various $d$ with $=1.0$

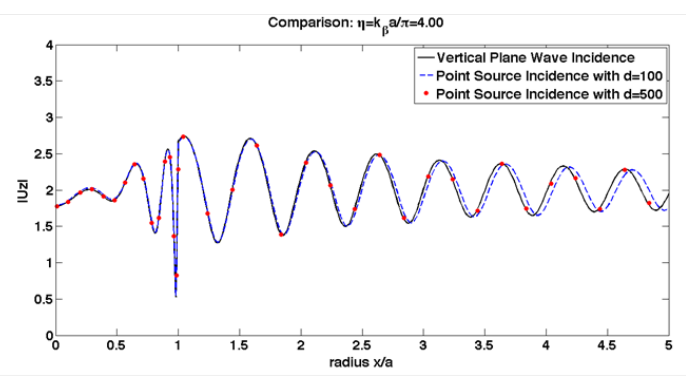

Figure 16: Vertical displacement amplitude along $\mathrm{x} / \mathrm{a}$ for various $\mathrm{d}$ with $=4.0$.

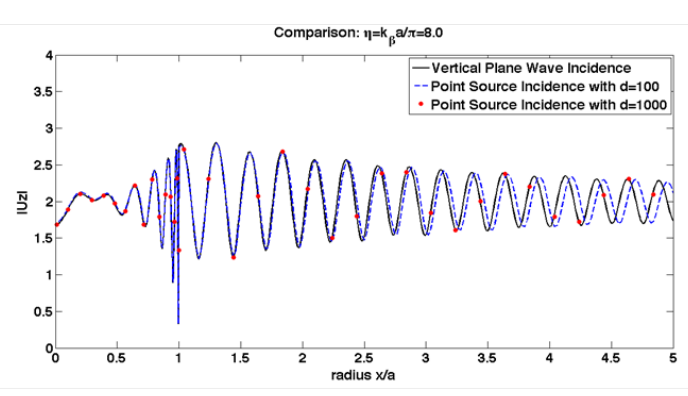

Figure 17: Vertical displacement amplitude along x/a for various $\mathrm{d}$ with $=8.0$. 
For this, when the frequency is not super high, the plane incidences can be used for the simulation of the point-sourced incidence assumed to be located deep such as $d=200$ a for $\eta$ $=1, \mathrm{~d}=500 \mathrm{a}$ for $\eta=4$, and $\mathrm{d}=1000 \mathrm{a}$ for $\eta=8$ which can guarantee the good matches along the radial distance up to 5 in these figure presented. Note that in all cases, the differences show up as phase shifts, while their amplitudes do agree.

\section{Conclusion}

This paper applies the series expansion of odd-order Legendre polynomials to the wave diffraction around a hemispherical canyon by a vertical point-sourced P-incidence. Results of various source depths and dimensionless frequencies are discussed. Following are the major aspects summarized for this problem:

1. The antisymmetric image method and the odd-orderonly Legendre polynomials can be effectively used to relax the zero-stress boundary conditions on the half-space. The calculable range of is high, although the highest selected in the discussion is 20 .

2. The oscillations are intensive near the rim of canyon, in particular for the case of high. So that the "dip and spike" effect also exists in the point-sourced problems.

3. A higher must result in a more complex oscillatory pattern on the surface of canyon, and the half-space. The same conclusion was already made for the normal plane P-incidence in the previous paper [1].

4. For incidence with a shallow epicenter, the substantial impacts can be caught on the canyon surface, while the influence on the surface of the half-space is relatively trivial and decays rapidly to zero.

5. For incidence with a deep epicenter, its impact on the half-space surface in the near range is comparable to that on the canyon surface. The deeper source and higher frequency both help extend the range of impact. However, the amplitudes will drop to zero at a far distance eventually as long as the incidence is point-sourced.

6. The statement in [2] "the results suggest that for the frequencies of interest in earthquake engineering, the plane-wave representation of incident SH waves will lead to reasonably realistic predictions of amplification of strongmotion amplitudes on the ground surface" still holds for the 3-D vertical P-wave incidence as well as for a larger range of the dimensionless frequencies.

\section{A. Mow and Pao (1973)}

The stress and displacement functions:

$$
\begin{aligned}
& \sigma_{r}: E_{11}^{(i)}\left(n, k_{\alpha} r\right)=\frac{2 \mu}{r^{2}}\left[\left(n^{2}-n-\frac{k_{\beta}^{2} r^{2}}{2}\right) z_{n}^{(i)}\left(k_{\alpha} r\right)+2 k_{\alpha} r z_{n+1}^{(i)}\left(k_{\alpha} r\right)\right] ; \quad \text { (A.1) } \\
& E_{1.3}^{(i)}\left(n, k_{\beta} r\right)=\frac{2 \mu}{r^{2}}\left\{n(n+1)\left[(n-1) z_{n}^{(i)}\left(k_{\beta} r\right)-k_{\beta} r z_{n+1}^{(i)}\left(k_{\beta} r\right)\right]\right\} ; \\
& \sigma_{\theta}: E_{21}^{(i)}\left(n, k_{\alpha} r\right)=\left(-n^{2}-\frac{k_{\beta}^{2} r^{2}}{2}+k_{\alpha}^{2} r^{2}\right) z_{n}\left(k_{\alpha} r\right)-k_{\alpha} r z_{n+1}\left(k_{\alpha} r\right) ;
\end{aligned}
$$

$$
\begin{gathered}
E_{23}^{(i)}\left(n, k_{\beta} r\right)=-\left(n^{2}+n\right)\left[n z_{n}\left(k_{\beta} r\right)-k_{\beta} r z_{n+1}\left(k_{\beta} r\right)\right] ; \\
\tau_{r \theta}: E_{41}^{(i)}\left(n, k_{\alpha} r\right)=\frac{2 \mu}{r^{2}}\left[(n-1) z_{n}^{(i)}\left(k_{\alpha} r\right)-k_{\alpha} r z_{n+1}^{(i)}\left(k_{\alpha} r\right)\right] ; \text { (A.5) } \\
E_{43}^{(i)}\left(n, k_{\beta} r\right)=\frac{2 \mu}{r^{2}}\left[\left(n^{2}-1-\frac{k_{\beta}^{2} r^{2}}{2}\right) z_{n}^{(i)}\left(k_{\beta} r\right)+k_{\beta} r z_{n+1}^{(i)}\left(k_{\beta} r\right)\right] ; \text { (A.6) } \\
U_{r}: D_{11}^{(i)}\left(n, k_{\alpha} r\right)=n z_{n}^{(i)}\left(k_{\alpha} r\right)-k_{\alpha} r z_{n+1}^{(i)}\left(k_{\alpha} r\right) ; \text { (A.7) } \\
\quad D_{13}^{(i)}\left(n, k_{\beta} r\right)=n(n+1) z_{n}^{(i)}\left(k_{\beta} r\right) ;(\text { A.8) } \\
U_{\theta}=D_{21}^{(i)}\left(n, k_{\alpha} r\right)=z_{n}^{(i)}\left(k_{\alpha} r\right) ;(\text { A.9) } \\
D_{23}^{(i)}\left(n, k_{\beta} r\right)=(n+1) z_{n}^{(i)}\left(k_{\beta} r\right)-k_{\beta} r z_{n+1}^{(i)}\left(k_{\beta} r\right) \text {. (A.10) }
\end{gathered}
$$

Where $z_{n}^{(i)}(),. \mathrm{i}=1,3$, are separately referred to the first kind of spherical-Bessel function with order $\mathrm{n} n j_{n}($.$) and first kind of$ Hankel function with order $n h_{n}^{(1)}($.$) .$

\section{B. Derivation of Scattered Waves for Normal Point-}

\section{Source P-wave Incidence}

This part is to prove the validity of the odd-term-only Legendre polynomials of the scattered P-and S-waves. The scattered waves by a hemispherical canyon in the half-space can be seen as the superposition of waves from two full space models of the spherical cavity: Model 0 and Model 1.

Model 0 is described in Figure B.1, that there is a source located below the cavity, on the positive $\mathrm{z}$-axis with a distance $\mathrm{d}$, emitting the P-wave radially. The P-wave is propagating on the $x-z$ plane and it interacts on the spherical cavity surface then generates the scattered P- and SV-waves. (Term $\exp (-i \omega t)$ is omitted in all equations for convenience.)

The potential of the incident $\mathrm{P}$-wave is given by $\varphi_{0}^{i}=\left\{\begin{array}{l}\sum_{n=0}^{\infty} i \varphi_{0} k_{\alpha}(2 n+1) h_{n}^{(1)}\left(k_{\alpha} d\right) j_{n}\left(k_{\alpha} r\right) P_{n}(-u), r<d, \\ \sum_{n=0}^{\infty} i \varphi_{0} k_{\alpha}(2 n+1) j_{n}\left(k_{\alpha} d\right) h_{n}^{(1)}\left(k_{\alpha} r\right) P_{n}(-u), r \geq d,\end{array}\right.$

Where $\mathrm{r}$ is the radius and $u=\cos \theta$ that is the polar angle of the spherical coordinates that is sharing the same origin with the Cartesian coordinates in Figure B.1.

The scattered waves due to presence of the spherical cavity can be expressed in the following form:

$$
\begin{gathered}
\varphi_{0}^{S}=\varphi_{0} \sum_{n=0}^{\infty} A_{n} h_{n}^{(1)}\left(k_{\alpha} r\right) P_{n}(u), \\
X_{0}^{S}=\varphi_{0} \sum_{n=0}^{\infty} C_{n} h_{n}^{(1)}\left(k_{\beta} r\right) P_{n}(u) .
\end{gathered}
$$

\section{(Figure B.1)}

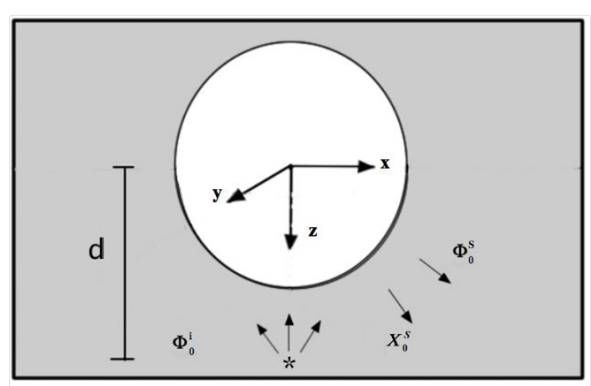

Figure B.1: Model 0: Upward point-source incident p-wave onto spherical cavity. 
Both expressions are independent of the spherical coordinate since the model is axisymmetric. Coefficients $A_{n}$ and $C_{n}$ can be solved exactly by the boundary conditions

$$
\sigma_{r}=\tau_{r \theta}=0
$$

At $\mathrm{r}=\mathrm{a}$ where is the surface of the spherical cavity. Thus $\varphi_{0}^{s}$ and $\chi_{0}^{s}$ form a complete solution of this wave problem that was already solved in the 1970s [8].

Similarly, a $2^{\text {nd }}$ full space model will be considered. Different from Model 0, Model 1 in Figure B.2 depicts a source right above the spherical cavity in the same way. A new Cartesian coordinate system is adopted here where,

$$
x_{1}=-x, \quad y_{1}=-y, \quad z_{1}=-z \text {. }
$$

\section{(Figure B.2)}

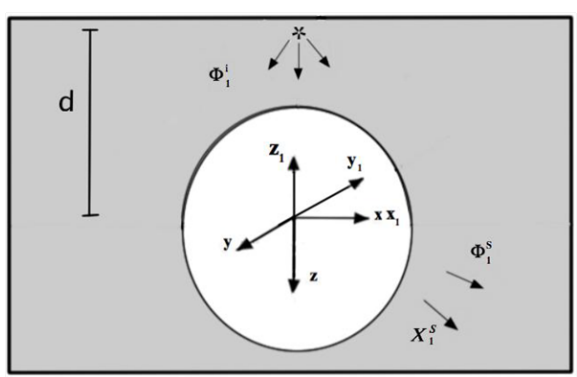

Figure B.2: Model 1: Downward point-source incident p-wave onto spherical cavity.

$\left(r_{1}, \theta_{1}, \phi_{1}\right)$ is the spherical coordinate system corresponding to the Cartesian coordinate system $\left(x_{1}, y_{1}, z_{1}\right)$. With $u_{1}=\cos \theta_{1}$, the incident P-wave potential $\varphi_{1}^{i}$ in Fig.B. 2 can be expressed as

$$
\varphi_{1}^{i}=\left\{\begin{array}{l}
\sum_{n=0}^{\infty} i \varphi_{1} k_{\alpha}(2 n+1) h_{n}^{(1)}\left(k_{\alpha} d\right) j_{n}\left(k_{\alpha} r_{1}\right) P_{n}\left(-u_{1}\right), r_{1}<d, \\
\sum_{n=0}^{\infty} i \varphi_{1} k_{\alpha}(2 n+1) j_{n}\left(k_{\alpha} d\right) h_{n}^{(1)}\left(k_{\alpha} r_{1}\right) P_{n}\left(-u_{1}\right), r \geq d,
\end{array}\right.
$$

With $\varphi_{1}$ as its amplitude. The resultant scattered wave potentials $\varphi_{1}^{s}$ and $\chi_{1}^{s}$ in the coordinate system $\left(r_{1}, \theta_{1}, \phi_{1}\right)$ are given by

$$
\begin{aligned}
& \varphi_{1}^{S}=\varphi_{1} \sum_{n=0}^{\infty} A_{n} h_{n}^{(1)}\left(k_{\alpha} r_{1}\right) P_{n}\left(u_{1}\right), \\
& X_{1}^{S}=\varphi_{0} \sum_{n=0}^{\infty} C_{n} h_{n}^{(1)}\left(k_{\beta} r\right) P_{n}\left(u_{1}\right) .
\end{aligned}
$$

Which are in the same form of $\varphi_{1}^{s}$ and $\chi_{1}^{s}$. The coefficients of the scattered potential are again completely solved because Model 1 can be taken as an adapted case of Model 0 . From the following relationships between coordinates $(r, \theta, \phi)$ and $\left(r_{1}, \theta_{1}, \phi_{1}\right)$ from (B.4) that

$$
\begin{aligned}
& r_{1}=r, \theta_{1}=\pi-\theta, \theta_{1}=2 \pi-\phi, \\
& \text { There is } u_{1}=\cos \theta_{1}=-\cos \theta=-u .
\end{aligned}
$$

And $\varphi_{1}^{s}$ and $\chi_{1}^{s}$ are then also given by, w.r.to. $(r, \theta, \phi)$ :

$$
\begin{aligned}
& \varphi_{1}^{S}=\varphi_{0}^{S}(r, \theta)=\varphi_{0} \sum_{n=0}^{\infty} A_{n} h_{n}^{(1)}\left(k_{\alpha} r\right)(-1)^{n} P_{n}(u), \\
& X_{1}^{S}=X_{0}^{S}(r, \theta)=\varphi_{0} \sum_{n=0}^{\infty} C_{n} h_{n}^{(1)}\left(k_{\beta} r\right)(-1)^{n} P_{n}(u), \\
& \text { with . } P_{n}\left(u_{1}\right)=P_{n}\left(-u_{1}\right)=(-1)^{n} P_{n}(u)
\end{aligned}
$$

The half-space model is next considered. Figure B.3 shows a hemispherical canyon with center located at the origin of the Cartesian coordinate $(x, y, z)$ that of the same description as before, and the entire $x-y$ plane $(z=0)$ is the half-space surface, with $\mathrm{z}>0$ the elastic half-space. A point-source P-wave with potential $\varphi^{i}$ vertically below the canyon will result in a pointsource P-wave with potential $\varphi^{r}$ vertically above the canyon, so that, from (B.1) and (B.5),

$$
\begin{gathered}
\varphi^{i}=\left\{\begin{array}{l}
\sum_{n=0}^{\infty} i \varphi_{0} k_{\alpha}(2 n+1) h_{n}^{(1)}\left(k_{\alpha} d\right) j_{n}\left(k_{\alpha} r\right) P_{n}(-u), r_{1}<d, \\
\sum_{n=0}^{\infty} i \varphi_{0} k_{\alpha}(2 n+1) j_{n}\left(k_{\alpha} d\right) h_{n}^{(1)}\left(k_{\alpha} r\right) P_{n}(-u), r \geq d,
\end{array}\right. \\
\text { w.r.t. }(r, \theta, \phi) \text { with origin at O(Figure B.1), and } \\
\varphi^{r}=\left\{\begin{array}{l}
\sum_{n=0}^{\infty} i \varphi_{1} k_{\alpha}(2 n+1) h_{n}^{(1)}\left(k_{\alpha} d\right) j_{n}\left(k_{\alpha} r_{1}\right) P_{n}\left(-u_{1}\right), r_{1}<d, \\
\sum_{n=0}^{\infty} i \varphi_{1} k_{\alpha}(2 n+1) j_{n}\left(k_{\alpha} d\right) h_{n}^{(1)}\left(k_{\alpha} r_{1}\right) P_{n}\left(-u_{1}\right), r \geq d .
\end{array}\right.
\end{gathered}
$$

w.r.t. $\left(r_{1}, \theta_{1}, \phi_{1}\right)$ with origin at O(Figure B.2), and

\section{(Figure B.3)}

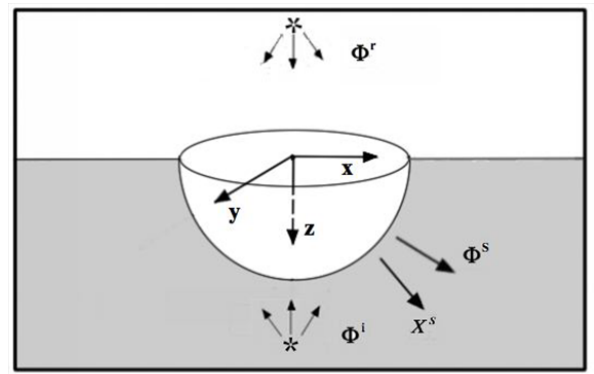

Figure B.3: Half-space model: Upward point-source incident p-wave onto hemispherical canyon.

With both $\varphi^{i}$ and $\varphi^{r}$, their superposition needs to satisfy the zero-stress half-space boundary conditions, then $\varphi_{1}=-\varphi_{0}$. Take $\varphi_{0}=1$ so that $\varphi_{1}=-1$. Then, as discussed in Section 3 above, the two point sources together with will satisfy the zero-stress half-space boundary conditions of (6). The half-space model in Figure B.3 can now be considered as a superposition of the models in Figure B.1 and Figure B.2 such that

$$
\begin{aligned}
& \varphi^{i} \text { of Half-Space Model }=\varphi_{0}^{i} \text { of Model } 0, \\
& \varphi^{r} \text { of Half-Space Model }=\varphi_{1}^{i} \text { of Model 1. (B.8) }
\end{aligned}
$$

In other words, the half-space model is the superposition of Model 0 and Model 1. The presence of the hemispherical canyon will result in the scattered wave's and , $\chi_{s}$ which can be taken as the superposition of the scattered waves of Model 0 and Model 1:

$$
\begin{aligned}
& \varphi^{s}=\varphi_{0}^{S}+\varphi_{1}^{S}, \\
& X^{s}=X_{0}^{S}+X_{1}^{S},
\end{aligned}
$$

Which takes the form, with $\varphi_{0}=1$ and $\varphi_{1}=-1$. 


\section{Civil Engineering Research Journal}

From the above equations

$$
\begin{aligned}
& \varphi^{s}=\varphi_{0}^{S}+\varphi_{1}^{S}=\sum_{n=0}^{\infty} A_{n} h_{n}^{(1)}\left(k_{\alpha} r\right) P_{n}(u)-\sum_{n=0}^{\infty} A_{n} h_{n}^{(1)}\left(k_{\alpha} r\right)(-1)^{n} P_{n}(u) \\
& =\sum_{n=0}^{\infty} A_{2 n+1}^{\prime} h_{(2 n+1)}^{(1)}\left(k_{\alpha} r\right) P_{2 n+1}(u),
\end{aligned}
$$

Which is with the odd-degree-only terms and $A_{2 n+1}^{\prime}=2 A_{2 n+1}$.

Similarly,

$$
\begin{aligned}
& \chi^{s}=\chi_{0}^{s}+\chi_{1}^{s}=\sum_{n=0}^{\infty} C_{n} h_{n}^{(i)}\left(k_{\beta} r\right) P_{n}(u)-\sum_{n=0}^{\infty} C_{n} h_{n}^{(1)}\left(k_{\beta} r\right)(-1)^{n} P_{n}(u) \\
& =\sum_{n=0}^{\infty} C_{2 n+1}^{\prime} h_{(2 n+1)}^{(i)}\left(k_{\beta} r\right) P_{2 n+1}(u),
\end{aligned}
$$

Which is also with the odd-degree-only terms and $C_{2 n+1}^{\prime}=2 C_{2 n+1}$ . Equations (B.11) and (B.12) are now the complete solution for the scattered waves to be used in the paper, (16) above, which has been shown to satisfy the zero-stress boundary conditions at the half-space surface. They are the superposition of the complete solutions from Model 0 and Model 1.

\section{References}

1. Lee VW, Zhu GY (2014) A note on three-dimensional scattering and diffraction by a hemispherical canyon-I: Vertically incident plane P-wave. Soil Dynamics and Earthquake Engineering 197-211.

2. Kara HF, Trifunac MD (2013) A note on plane-wave approximation. Soil Dynamics and Earthquake Engineering 51: 9-13.

3. Alterman ZS, Aboudi J, Karal FC (1970) Pulse propagation in a laterally heterogeneous solid elastic sphere. Geophysical Journal International 21(3): 243-260.

4. Tadeu A, Santos P, Ant'onio J (2001) Amplification of elastic waves due to a point source in the presence of complex surface topography. Computers and Structures 79(18): 1697-1712.

5. Alterman ZS, Karal FC (1968) Propagation of elastic waves in layered media by finite difference methods. Bulletin of the Seismological Society of America 58(1): 367-398.

6. Dassios G, Hadjinicolaou M, Kamvyssas G (1999) Direct and inverse scattering for point source fields: The penetrable small sphere. ZAMMJournal of Applied Mathematics and Me-chanics/Zeitschrift f ur Angewandte Mathematik und Mechanik 79(5): 303-316.

7. Rudnick I (1947) The propagation of an acoustic wave along a boundary. The Journal of the Acoustical Society of America 19(2): 348356.

8. Mow CC, Pao YH (1971) The diffraction of elastic waves and dynamic stress concentrations. Rand Corporation, Santa Monica.

9. Abramowitz M, Stegun IA (1972) Handbook of mathematical functions: with formulas, graphs, and mathematical tables. Courier Dover Publications, USA.

10. Lee VW, Trifunac MD (1979) Response of tunnels to incident SH-waves. Journal of the Engineering Mechanics Division 105(4): 643-659.

11. Trifunac MD (1972) Scattering of plane SH-waves by a semi-cylindrical canyon Earthquake Engineering and Structural Dynamics 1(3): 267281.

\section{Your next submission with Juniper Publishers will reach you the below assets}

- Quality Editorial service

- Swift Peer Review

- Reprints availability

- E-prints Service

- Manuscript Podcast for convenient understanding

- Global attainment for your research

- Manuscript accessibility in different formats

( Pdf, E-pub, Full Text, Audio)

- Unceasing customer service

Track the below URL for one-step submission https://juniperpublishers.com/online-submission.php 